\title{
A CODIMENSION THEOREM FOR PSEUDO-NOETHERIAN RINGS
}

BY

\author{
KENNETH MCDOWELL
}

ABSTRACT. M. Auslander and M. Bridger have shown that the depth of a Noetherian local ring is the sum of the Gorenstein dimension and the depth of any given nonzero finitely generated module of finite Gorenstein dimension. In this paper it is demonstrated that this result remains true when suitably interpreted for the class of coherent rings herein entitled pseudoNoetherian rings. This class contains, among others, all Noetherian rings and valuation domains as well as non-Noetherian local rings of infinite depth.

1. Introduction. In a paper entitled Stable module theory, M. Auslander and M. Bridger introduce the concept of Gorenstein dimension (denoted G-dim) for finitely generated modules over a commutative Noetherian ring and, after lengthy homological considerations, arrive at the following codimension theorem.

THEOREM [1, THEOREM 4.13]. Let $(R, m)$ be a local Noetherian ring and $M$ a nonzero finitely generated $R$-module with finite Gorenstein dimension. Then $($ G-dim $M)+(\operatorname{depth} M)=\operatorname{depth} R$.

This result is a generalization of the original codimension theorem for Noetherian rings and finitely generated modules of finite projective dimension (cf. [6, Theorem 173]). In [7] the following concept is introduced.

DEFINITION. A commutative ring $R$ is called pseudo-Noetherian if it satisfies the following two conditions.

(1) $R$ is coherent.

(2) If $M$ is a nonzero finitely presented $R$-module and $I$ is a finitely generated ideal of $R$ contained in the zero-divisors of $M$, then there exists a nonzero $m$ in $M$ with $I m=0$.

The depth of a finitely presented module has a suitable interpretation over a local pseudo-Noetherian ring, and Gorenstein dimension may be defined for finitely presented modules over coherent rings in exactly the same way as it is defined for finitely generated modules over Noetherian rings. Many theorems of a homological nature which are true for finitely generated modules over a Noe-

Received by the editors August 5, 1974.

AMS (MOS) subject classifications (1970). Primary 13C99; Secondary 13D05, 13 A99.

Key words and phrases. Coherent ring, projective dimension, Gorenstein dimension, depth of a module.

Copyright $\odot 1975$, American Mathematical Society 
therian ring possess analogues in the theory of finitely presented modules over a pseuda-Noetherian ring (see [7]). This paper demonstrates that the codimension theorem mentioned above is of this type.

Because the proofs of many of the results of [1] depend primarily on the fact that finitely generated modules over Noetherian rings have finitely generated projective resolutions, such proofs also apply, with only minor alterations, to the coherent case. (Finitely presented modules over coherent rings have finitely generated projective resolutions.) Hence, certain results of [1] shall be freely employed without proof here in the coherent situation even though they were originally presented in the Noetherian context.

2. Preliminaries. All rings are commutative with unit and all modules unitary. $\mathbf{Z}$ will denote the integers and $\mathbf{Q}$ the rational numbers. $(R, \mathrm{~m})$ will denote a local not necessarily Noetherian ring $R$ with unique maximal ideal $\mathrm{m}$. For a module $M$ over a ring $R$, the set of all elements of $R$ which have nonzero annihilator in $M$ will be denoted by $Z(M)$. The dual module $\operatorname{Hom}_{R}(M, R)$ will be denoted by $M^{*}$. A ring $R$ is called coherent if each of its finitely generated ideals is finitely presented. (Further information on coherent rings may be found in [3] and [5].)

Suppose $M$ is a finitely presented module over a coherent ring $R$ and choose a finitely generated projective resolution for $M$ as follows:

$$
\cdots \rightarrow P_{i+1} \rightarrow P_{i} \rightarrow P_{i-1} \rightarrow \cdots \rightarrow P_{1} \rightarrow P_{0} \rightarrow M \rightarrow 0 \text {. }
$$

Then $\operatorname{ker}\left(P_{i-1} \rightarrow P_{i-2}\right)(i>1)$ is called the $i$ th syzygy of $M$ and is denoted $\Omega^{i}(M) . \Omega^{0}(M)=M$ and $\Omega^{1}(M)=\operatorname{ker}\left(P_{0} \rightarrow M\right)$. Coker $\left(P_{0}^{*} \rightarrow P_{1}^{*}\right)$ is denoted $D M$ and with this, $0 \rightarrow M^{*} \rightarrow P_{0}^{*} \rightarrow P_{1}^{*} \rightarrow D M \rightarrow 0$ is an exact sequence. Note that $D M, \Omega^{i}(M)$, and $M^{*}$ are again finitely presented modules. (It does not matter which projective resolution we use for $M$ since $D M$ and $\Omega^{i}(M)$ are determined up to projective equivalence by the projective equivalence class of $M$ [ 1 , Corollary 2.3 and remark, p. 53].)

For the definition of $R$-sequence and a description of the relevance of this concept to the theory of pseudo-Noetherian rings, the reader may wish to consult [7] in which he may also find various examples of local pseudo-Noetherian rings.

3. Gorenstein dimension. $\left({ }^{1}\right)$ A functor is called projectively (injectively) stable if it vanishes on projectives (injectives). Gorenstein dimension is defined in terms of the projective or injective stability of certain functors. The definition may at first seem rather complex, but it will be seen that there is a much simpler description for modules of finite Gorenstein dimension. Indeed, it is the finitely presented modules

(1) $\$ 3$ is expository in nature; all of this material was originally presented for the Noetherian situation in [1]. These results are, however, needed in $\$ 4$ and are included here for the sake of convenience, conciseness and completeness. 
of finite dimension which are important in the later results.

Throughout this section, $R$ will denote a coherent ring and $M$ a finitely presented $R$-module. If $k$ is a nonnegative integer, $\operatorname{Ext}_{R}^{i}\left(\Omega^{k} M,\right)$ is projectively stable for all $i>0$, and $\operatorname{Tor}_{i}^{R}\left(D \Omega^{k} M\right.$, ) is injectively stable for all $i>0$, then, for any $n \geqslant k, \operatorname{Ext}_{R}^{i}\left(\Omega^{n} M,\right)$ is projectively stable for all $i>0$ and $\operatorname{Tor}_{i}^{R}\left(D \Omega^{n} M,\right)$ is injectively stable for all $i>0$.

3.1 Definition. Gorenstein dimension $M$ equals $\inf \left\{k \mid \operatorname{Ext}_{R}^{i}\left(\Omega^{k} M,\right)\right.$ is projectively stable for all $i>0$ and $\operatorname{Tor}_{i}^{R}\left(D \Omega^{k} M, \quad\right)$ is injectively stable for all $i>0$ \}. Denote Gorenstein dimension $M$ by G-dim $R_{R} M$ or simply G-dim $M$.

3.2 RemarK. If $\mathrm{G}-\operatorname{dim} M<\infty$ then $\mathrm{G}-\operatorname{dim} M=\sup \left\{t \mid \operatorname{Ext}_{R}^{t}(M, R) \neq 0\right\}$. In fact, if G-dim $M<\infty, \mathrm{G}-\operatorname{dim} M=\sup \left\{t \mid \operatorname{Ext}_{R}^{t}(M\right.$,$) is not projectively$ stable\} [1, Corollary 3.6]. Now $\operatorname{Ext}_{R}^{n}(M$,$) commutes with directed colimits for$ all $n \geqslant 0 \quad$ [8, Theorem 3.2] and every projective module is a direct summand of a free module. Hence, $\operatorname{Ext}_{R}^{n}(M$,$) is projectively stable if and only if$ $\operatorname{Ext}_{R}^{n}(M, R)=0$.

3.3 REMARK. If $\mathrm{G}$-dim $M=0, M$ is reflexive.

In fact, if G-dim $M=0$, Definition 3.1 shows that $\operatorname{Tor}_{i}^{R}(D M$,$) is injectively$ stable for all $i>0$. Hence,

$$
\operatorname{Hom}_{\mathrm{Z}}\left(\operatorname{Ext}_{R}^{i}(D M, P), \mathrm{Q} / \mathrm{Z}\right) \cong \operatorname{Tor}_{i}^{R}\left(D M, \operatorname{Hom}_{\mathrm{Z}}(P, \mathrm{Q} / \mathrm{Z})\right)=0
$$

for any projective $P$ and any $i>0\left[4\right.$, p. 120]. This shows $\operatorname{Ext}_{R}^{i}(D M$,$) is pro-$ jectively stable. Since there exists an exact sequence

$$
0 \rightarrow \operatorname{Ext}_{R}^{1}(D M,) \rightarrow M \otimes_{R} \longrightarrow \operatorname{Hom}_{R}\left(M^{*},\right) \rightarrow \operatorname{Ext}_{R}^{2}(D M,)
$$

\section{[1, Theorem 2.8], $M$ is clearly reflexive.}

3.4 RemarK. Denote the projective dimension of $M$ by p.dim $M$. G-dim $M$ $\leqslant \mathrm{p} \cdot \operatorname{dim} M$ and if p. $\operatorname{dim} M<\infty, \mathrm{G}-\operatorname{dim} M=\mathrm{p} \cdot \operatorname{dim} M$.

In fact, for any nonnegative integer $k, \mathrm{G}-\operatorname{dim} M \leqslant k$ if and only if there exists an exact sequence of finitely presented modules, $0 \rightarrow X_{k} \rightarrow \cdots \rightarrow X_{1}$ $\rightarrow X_{0} \rightarrow M \rightarrow 0$, with the property that G-dim $X_{i}=0$ for $0 \leqslant i \leqslant k[1$, Theorem 3.13]. Hence, since the Gorenstein dimension of projective modules is zero, $\mathrm{G}-\operatorname{dim} M \leqslant \mathrm{p} . \operatorname{dim} M$. If p. $\operatorname{dim} M=k<\infty, \operatorname{Ext}_{R}^{k}(M, R) \neq 0$ [4, Chapter VI, Exercise 9] and, therefore, G-dim $M=k$ by Remark 3.2.

The following proposition demonstrates that projective dimension and Gorenstein dimension are alike in their behaviour. (As usual, $R$ denotes a coherent ring and $M$ a finitely presented module.)

3.5 Proposition (a) (cf. [1, Corollary 3.15]). If G-dim $M \neq 0$ and $0 \rightarrow N \rightarrow X \rightarrow M \rightarrow 0$ is an exact sequence of finitely presented $R$-modules with $\mathrm{G}-\operatorname{dim} X=0$, then $\mathrm{G}-\operatorname{dim} N=\mathrm{G}-\operatorname{dim} M-1$.

(b) (cf. [1, Corollary 4.30], Third Change of Rings Theorem for Goren- 
stein Dimension). Assume $(R, \mathrm{~m})$ is a local coherent ring and $x \in \mathfrak{m}$ is a

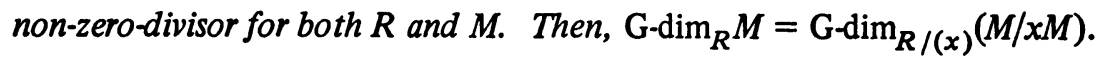

(c) (cf. [1, Lemma 4.32], First Change of Rings Theorem for Gorenstein Dimension). Assume $(R, \mathrm{~m})$ is a local coherent ring, $M$ is a nonzero finitely presented module, and $x$ is a non-zero-divisor of $R$ which annihilates $M$. Then, if $\mathrm{G}-\operatorname{dim}_{R /(x)} M=k<\infty, \mathrm{G}-\operatorname{dim}_{R} M=k+1$.

3.6 RemarK. If $(R, \mathrm{~m})$ is a local coherent ring, $M \neq 0, \mathrm{G}-\operatorname{dim} M<\infty$, and $x \in \mathrm{m}$ is a non-zero-divisor for both $R$ and $M$, then parts (b) and (c) of the above proposition show that $\mathrm{G}-\operatorname{dim}_{R}(M / x M)=\mathrm{G}-\operatorname{dim}_{R} M+1$.

4. The codimension theorem. Throughout this section $R$ will denote a local pseudo-Noetherian ring with maximal ideal $\mathfrak{m}$ and $M$ will denote a nonzero finitely presented $R$-module. Suppose $I$ is a proper finitely generated ideal of $R$. If there exists some integer $n$ such that $\operatorname{Ext}_{R}^{n}(R / I, M) \neq 0$, define

$$
R / I \text {-depth } M=\inf \left\{m \mid \operatorname{Ext}_{R}^{m}(R / I, M) \neq 0\right\} .
$$

Otherwise, $R / I$-depth $M=\infty$. In [7] it is shown that if $I$ and $J$ are two proper finitely generated ideals of $R$ with $I \subseteq J$, then $R / I$-depth $M \leqslant R / J$-depth $M$.

4.1 Definition. depth ${ }_{R} M$ (depth $M$ where no confusion arises) will denote $\sup \{R / I$-depth $M \mid I$ a finitely generated proper ideal of $R\}$.

In [7] it is demonstrated that depth $M$ is equal to the length of any maximal $M$-sequence. From this it follows that if $x \in \mathfrak{m}$ is a non-zero-divisor for $M$, then $\operatorname{depth}_{R /(x)}(M / x M)=\operatorname{depth}_{R}(M / x M)=\operatorname{depth}_{R} M-1$. Notice also that if $I$ is a proper finitely generated ideal of $R, R / I$ is again a local pseudo-Noetherian ring and $M / I M$ is a nonzero finitely presented module over this ring.

4.2 Proposition. Let $(R, m)$ be a local pseudo-Noetherian ring of finite depth and let $M$ be nonzero finitely presented $R$-module of finite Gorenstein dimension. The following statements are equivalent.

(a) $\mathrm{G}-\operatorname{dim} M=0$.

(b) depth $M \geqslant \operatorname{depth} R$.

(c) depth $M=\operatorname{depth} R$.

Proof. (a) implies (b). The methods of [1, Proposition 4.12] suffice to show that every $R$-sequence is an $M$-sequence. By the above remark, this means depth $M \geqslant \operatorname{depth} R$.

(b) implies (c). This is proven by induction on depth $R$. Therefore, suppose first that depth $R=0$. Assume by way of contradiction that $m$ contains an element $r \notin Z(M)$.

Suppose $I$ is a finitely generated ideal in $m$ containing $r$. Since depth $R=0$, $\mathrm{m}=Z(R)$ and because $R$ is pseudo-Noetherian, there exists $s \neq 0$ in $R$ with 
$r \in I \subseteq \operatorname{ann}_{R}(s)=s^{\circ}$. Now $\left(R / s^{\circ}\right) \cong s R \subseteq R$ and by applying $\operatorname{Hom}_{R}\left(M^{*},\right)$ to this, one obtains that $U \rightarrow \operatorname{Hom}_{R}\left(M^{*}, R / s^{\circ}\right) \rightarrow M^{* *}$ is exact. Since depth $R=0$, Theorem 5.4 of [2] shows that for any finitely presented module $N, N=0$ if and only if $N^{*}=0$. This fact may be used (as in Proposition 4.11 of [1]) to show that since G-dim $M<\infty, \mathrm{G}-\operatorname{dim} M=0$. Hence, by Remark $3.3, M^{* *} \cong M$. Now because $r \notin Z(M)$ and $r$ annihilates $\operatorname{Hom}_{R}\left(M^{*}, R / s^{\circ}\right), \operatorname{Hom}_{R}\left(M^{*}, R / s^{\circ}\right)=0$.

For each finitely generated ideal $I \subseteq \mathfrak{m}$ with $r \in I$, such an $s^{\circ}$ may be constructed with the above properties. Let $A$ represent the set of all these annihilators. Clearly $A$ is a cofinal set in the directed set of all finitely generated ideals in $\mathrm{m}$ and, therefore, $\lim _{\rightarrow} s^{\circ} \in A\left(R / s^{\circ}\right)=R / \mathrm{m}$. Furthermore, since $M$ is finitely presented and $R$ is coherent, $M^{*}$ is finitely presented [8, Lemma 4.7]. Hence $\operatorname{Hom}_{R}\left(M^{*},\right)$ commutes with directed colimits yielding $\operatorname{Hom}_{R}\left(M^{*}, R / \mathrm{m}\right)=0$. The natural surjection $M^{*} \rightarrow\left(M^{*} / \mathrm{m}^{*}\right)$ induces an injection

$$
\operatorname{Hom}_{R}\left(M^{*} / \mathrm{m} M^{*}, R / \mathrm{m}\right) \rightarrow \operatorname{Hom}_{R}\left(M^{*}, R / \mathrm{m}\right)=0 .
$$

Thus, $M^{*} / \mathrm{m}^{*}$ is zero and by Nakayama's lemma $M^{*}=0$. Since $M$ is reflexive, $M=0$. This contradiction shows that depth $M=0$ as required.

Now suppose depth $R=n \geqslant 1$ and the result holds for rings of depth less than $n$. By assumption, depth $M \geqslant \operatorname{depth} R=n$.

Claim. There exists $x$ in $m$ with $x \notin Z(R)$ and $x \notin Z(M)$.

In fact, suppose to the contrary that $\mathrm{m} \subseteq Z(R) \cup Z(M)$. Let $I$ be a finitely generated ideal contained in $\mathrm{m}$. Since $R$ is pseudo-Noetherian, $R \oplus M$ is a finitely presented $R$-module, and $I \subseteq Z(R) \cup Z(M)=Z(R \oplus M)$, there exists $(r, m) \in$ $R \oplus M$ with $r \neq 0$ or $m \neq 0$ such that $I(r, m)=0$. Therefore, $I r=0$ and $I m=0$ giving $I \subseteq Z(R)$ or $I \subseteq Z(M)$. Since depth $R \neq 0$, there exists a non-zero-divisor $y$ in $m$ and, hence, every finitely generated proper ideal $J$ containing $y$ is contained in $Z(M)$. Therefore, depth $M=0$. This contradiction establishes the claim.

For such an $x \in \mathfrak{m}$, Proposition 3.5(b) shows G-dim $\operatorname{di(x)}_{(M / x M)<\infty}$. By the induction assumption,

$$
\operatorname{depth}_{R /(x)}(M / x M)=\operatorname{depth}_{R /(x)}(R /(x))=\operatorname{depth} R-1 .
$$

Since depth $\operatorname{de}_{R /(x)}(M / x M)=\operatorname{depth}_{R} M-1$, depth $M=\operatorname{depth} R$ as required.

(c) implies (a). This is also proven by induction on depth $R$. If $\operatorname{depth} R=0$, G-dim $M=0$, again because $\mathrm{G}-\operatorname{dim} M<\infty$. Now suppose depth $R \geqslant 1$ and the result holds for rings of smaller depth. Since depth $M=\operatorname{depth} R \geqslant 1$, there exists $x \in \mathfrak{m}$ as above with $x \notin Z(R)$ and $x \notin Z(M)$. depth $M=\operatorname{depth} R$ implies (1) holds. Since G-dim $R /(x)(M / x M)=G-\operatorname{dim}_{R} M<\infty$ by Proposition 3.5(b), $\operatorname{Ext}_{R /(x)}^{i}(M / x M, R /(x))=0$ for all $i>0$ by the induction hypothesis and Remark 3.2. [4, Proposition 4.1.3, p. 118] illustrates that

$$
\operatorname{Ext}_{R}^{i}(M, R /(x)) \cong \mathrm{Ext}_{R /(x)}^{i}(M / x M, R /(x))=0
$$


for all $i \geqslant 0$. From the short exact sequence $0 \rightarrow R \stackrel{x}{\longrightarrow} R \rightarrow R /(x) \rightarrow 0$, one obtains the exact sequence

$$
\operatorname{Ext}_{R}^{i}(M, R) \rightarrow \operatorname{Ext}_{R}^{i}(M, R) \rightarrow \operatorname{Ext}_{R}^{i}(M, R /(x)) .
$$

For all $i>0$ the last term is zero, giving $\operatorname{Ext}_{R}^{i}(M, R)=(x) \operatorname{Ext}_{R}^{i}(M, R)$. Since $\operatorname{Ext}_{R}^{i}(M, R)$ is finitely presented, Nakayama's lemma shows $\operatorname{Ext}_{R}^{i}(M, R)=0$ for all $i>0$. By Remark 3.2, G-dim $M=0$.

The following lemma is employed in the proof of the main result.

4.3 LEMmA. Let $(R, \mathfrak{m})$ be a local pseudo-Noetherian ring and $M$ a nonzero finitely presented $R$-module. Furthermore, suppose depth $M=0$ and $0 \rightarrow K \rightarrow$ $F \rightarrow M \stackrel{\nu}{\longrightarrow} 0$ is a finite presentation of $M$ with $F$ finitely generated free and $K$ finitely generated. Then if $x$ is a non-zero-divisor in $\mathrm{m}, \operatorname{depth}_{R /(x)}(K / x K)=0$.

Proof. It is sufficient, by Definition 4.1, to show that for every finitely generated ideal $I$ with $x \in I \subseteq \mathrm{m}, R / I$-depth $\operatorname{de}_{(x)}(K / x K)=0$. Observe that depth $M=0$ and therefore for such an $I, I \subseteq Z(M)$. Because $R$ is pseudo-Noetherian, there exists a nonzero $m$ in $M$ with $I m=0$. Let $u \in F$ with $v(u)=m$. Now $u \notin K, I u \subseteq K$ and $x \notin Z(K)$. Therefore $x u \notin x K, x u \in K$ and $I x u \subseteq x K$. Let $\mu: K \rightarrow K / x K$ be the natural surjection. Then $\mu(x u) \neq 0$ and $I \mu(x u)=0$. Hence, $\mu(x u)$ induces a nonzero homomorphism in $\operatorname{Hom}_{R /(x)}(R / I, K / x K)$. This means $R / I$-depth ${ }_{R /(x)}(K / x K)=0$ as required. (This proof is essentially contained in the proof of Theorem 173 of [6].)

4.4 THEOREM. Let $(R, m)$ be a local pseudo-Noetherian ring and let $M$ be a nonzero finitely presented $R$-module with finite Gorenstein dimension. Then,

$$
(\text { G-dim } M)+(\operatorname{depth} M)=\operatorname{depth} R \text {. }
$$

Proof. Case 1. depth $R<\infty$.

Proceed by induction on G-dim $M$. If $\mathrm{G}-\operatorname{dim} M=0$, depth $M=\operatorname{depth} R$ by Proposition 4.2. Now suppose G-dim $M=n>0$ and let $0 \rightarrow K \rightarrow P \rightarrow M \rightarrow 0$ be a finite presentation for $M$ in which $P$ is finitely generated projective and $K$ is finitely generated. Since G-dim $M>0$, Proposition 4.2 indicates that depth $M<$ depth $P=\operatorname{depth} R$. With this it is easily proven that depth $K=\operatorname{depth} M+1$. Now G-dim $K=n-1$ by Proposition 3.5(a) and therefore, by the induction assumption,

$$
n-1=\mathrm{G}-\operatorname{dim} K=\operatorname{depth} R-\operatorname{depth} K=\operatorname{depth} R-\operatorname{depth} M-1 \text {. }
$$

Thus, $n=\mathrm{G}-\operatorname{dim} M=\operatorname{depth} R-\operatorname{depth} M$ as required.

Case 2. depth $R=\infty$

First, it will be shown that depth $M \neq 0$ by means of induction on G-dim $M$. The assumption depth $R<\infty$ is not needed in the proof of (a) implies (b) 
in Proposition 4.2. Hence, if $\mathrm{G} \cdot \operatorname{dim} M=0$, depth $M=\infty$. Now assume G-dim $M$ $=n>0$ and let $0 \rightarrow K \rightarrow F \stackrel{\nu}{\longrightarrow} M \rightarrow 0$ be a finite presentation of $M$ with $F$ finitely generated free and $K$ finitely generated. Since depth $R>0$, there exists a non-zero-divisor $x$ in $m$ which is clearly also a non-zero-divisor for $K$. Now $K / x K$ is a finitely presented $R /(x)$-module with the property that G-dim $R /(x) K / x K=$ G-dim $\operatorname{dim}_{R} K=n-1$ (Proposition 3.5(a), (b)). Furthermore, $R /(x)$ is a local pseudoNoetherian ring of infinite depth. Hence, by the induction assumption, $\operatorname{depth}_{R /(x)}(K / x K) \neq 0$. Lemma 4.3 shows that depth $M \neq 0$.

Now suppose by way of contradiction that depth $M<\infty$. Since depth $M$ and depth $R$ are both nonzero, there exists an $x$ in $m$ which is a non-zero-divisor for both $R$ and $M$. (See proof of (b) implies (c), Proposition 4.2). By Remark 3.6, $\operatorname{G}_{-\operatorname{dim}_{R}}(M / x M)=\mathrm{G}-\operatorname{dim}_{\mathrm{R}} M+1$. Moreover, $\operatorname{depth}_{R}(M / x M)=\operatorname{depth}_{R} M-1$. Thus, $M / x M$ is a new nonzero finitely presented $R$-module with finite Gorenstein dimension but of smaller depth than $M$. Continuing in this manner, a finitely presented module with finite Gorenstein dimension may be found which has zero depth. This contradiction shows depth $M=\infty$.

It is clear by Remark 3.4 that Theorem 4.4 generalizes the original codimension theorem for Noetherian rings (cf. [6, Theorem 173]). A Noetherian ring is admittedly pseudo-Noetherian by virtue of the pleasant properties of the associated prime ideals. However, as far as the theory presented above is concerned, no reference to prime ideals is needed once the pseudo-Noetherian condition is assumed.

\section{REFERENCES}

1. M. Auslander and M. Bridger, Stable module theory, Mem. Amer. Math. Soc. No. 94 (1969). MR 42 \#4580.

2. H. Bass, Finitistic dimension and a homological generalization of semi-primary rings, Trans. Amer. Math. Soc. 95 (1960), 466-488. MR 28 \#1212.

3. N. Bourbaki, Éléments de mathématique. Fasc. XXVII-XXI. Algèbre commutative. Chaps. 1-7, Actualités Sci. Indust., nos. 1290, 1308, 1314, Hermann, Paris, 1961. MR 30 \#2027; 33 \#2660; 36 \# 46; 41 \#5339.

4. H. Cartan and S. Eilenberg, Homological algebra, Princeton Univ. Press, Princeton, N. J., 1956. MR 17, 1040.

5. S. U. Chase, Direct products of modules, Trans. Amer. Math. Soc. 97 (1960), 457473. MR 22 \#11017.

6. I. Kaplansky, Commutative rings, Allyn and Bacon, Boston, Mass., 1970. MR 40 \#7234.

7. K. McDowell, Pseudo-Noetherian rings, Canad. Math. Bull. (to appear).

8. B. T. Stenstrom, Coherent rings and FP-injective modules, J. London Math. Soc. (2) 2 (1970), 323-329. MR 41 \#3533.

\section{TARIO, CANADA}

DEPARTMENT OF MATHEMATICS, MCMASTER UNIVERSITY, HAMILTON, ON-

Current address: Department of Mathematics, Wilfrid Laurier University, Waterloo, Ontario, Canada 